\title{
Caractérisation de la diversité et de la durabilité des exploitations laitières familiales au Vietnam : étude de cas en zone périurbaine de Hanoï
}

\author{
Duy Khanh Pham ${ }^{1 *}$ Guillaume Duteurtre ${ }^{2}$ Sylvie Cournut ${ }^{3}$ \\ Samir Messad ${ }^{2}$ Benoît Dedieu ${ }^{4}$ Nathalie Hostiou ${ }^{5}$
}

\section{Mots-clés}

Exploitation laitière, diversification, intensification, typologie, Viêt Nam

Submitted: 2 May 2016

Accepted: 17 January 2017

Published: 27 June 2017

DOI: $10.19182 /$ remvt.31198

\begin{abstract}
Résumé
Au Vietnam, les politiques publiques tendent à soutenir le développement des exploitations laitières de grande taille familiales (> 30 vaches) ou industrielles (> 500 vaches). Cependant, malgré un essor récent de ces grandes fermes, les exploitations familiales de très petite taille continuent à jouer un rôle important dans la production nationale de lait. Pour contribuer à la réflexion sur les modèles de fermes à promouvoir, notre étude propose de caractériser la diversité des exploitations laitières présentes dans le district de Ba Vi en zone périurbaine de Hanoi et d'évaluer leur durabilité. Au total 160 exploitations laitières ont été sélectionnées pour représenter la diversité des fermes présentes dans la zone. Des informations ont été recueillies lors d'entretiens directifs avec les éleveurs sur les divers types de capitaux disponibles sur l'exploitation, sur les pratiques d'élevage et de commercialisation du lait, et sur leurs performances économiques, environnementales et sociales. Une analyse factorielle multiple, suivie d'une classification ascendante hiérarchique ont permis de réaliser une typologie des exploitations. En plus d'une ferme industrielle présente dans le district, cinq types d'exploitations laitières familiales ont été identifiés, qui se différenciaient surtout par le nombre et le type d'activités agricoles et non-agricoles, la taille du troupeau laitier, et l'intensification de la production laitière. Les indicateurs de performances des exploitations spécialisées ont révélé une rentabilité économique plus élevée que dans les exploitations plus diversifiées. Cependant, les exploitations mixtes avaient également des atouts sur les plans économique (souplesse d'adaptation), environnemental (autonomie fourragère) et social (emploi, charge de travail), qui étaient liés à la diversité de leurs activités agricoles et non-agricoles. Si les politiques publiques tendent à soutenir le développement des fermes spécialisées et des grandes fermes, les programmes d'appui doivent également concerner les petites exploitations associant activités agricoles et non-agricoles, car elles ont aussi un rôle majeur à jouer dans le développement durable de la production laitière nationale.
\end{abstract}

- Pour citer cet article : Pham Duy Khanh, Duteurtre G., Cournut S., Messad S., Dedieu B., Hostiou N., 2016. Characterizing dairy farm diversity and sustainability in Vietnam: Case study in suburban Hanoi. Rev. Elev. Med. Vet. Pays Trop., 69 (4): 131-141, doi: 10.19182/remvt.31198
1. Rudec-Ipsard, 16 Thuy Khue, Tay Ho, Hanoï, Vietnam.

2. Cirad, UMR Selmet, Montpellier, France.

3. VetAgroSup, UMR 1273 Métafort - VetAgro Sup, Lempdes, France.

4. INRA, Saint Genès Champanelle, France.

5. INRA, UMR 1273, Métafort, Lempdes, France.

* Auteur pour la correspondance

Tél. : 0473982915 ; email : khanh.rudec@gmail.com

(c) 0 https://creativecommons.org/licenses/by/4.0/

\section{INTRODUCTION}

Dans un contexte international marqué par l'augmentation de la spécialisation et de l'intensification de la production du lait, le Vietnam est questionné sur la durabilité de son tissu productif laitier qui s'appuie essentiellement sur des exploitations familiales (Hemme et Otte, 2010). Le Vietnam est un pays agricole en transition, avec une population de 89,7 millions d'habitants dont $68 \%$ vivent dans les zones rurales (OECD, 2015). L'agriculture joue un rôle important dans le revenu des ménages. En effet, la production agricole se base encore largement sur des petites exploitations familiales qui représentent 9,6 millions de ménages en 2011 avec 0,8 hectare en moyenne (GSO, 2014). Le secteur de l'élevage occupe une place importante dans le développement agricole, en participant pour $24,6 \%$ du produit intérieur brut agricole. 
La production laitière s'est développée de manière significative à partir des années 2000, contrairement à l'élevage de porcs et de volailles qui constitue une activité ancienne (Duteurtre et al., 2015a). Comme dans la plupart des pays d'Asie du Sud-Est, au Vietnam la consommation de lait est restée longtemps très faible mais elle connaît depuis plusieurs années une croissance rapide du fait de l'augmentation de la population urbaine et des changements des modes de consommation (Beghin, 2006). Pour répondre à cette demande, le gouvernement a initié au début des années 2000 un Programme national de développement laitier. De 2001 à 2013, la production laitière a connu un taux de croissance extrêmement élevé, avec une augmentation des troupeaux de 13,4\% par an et une croissance de la production de $17 \%$ par an (OECD, 2015). Cette croissance s'est appuyée sur l'essor de la production familiale. Sur les 20000 exploitations laitières présentes à la fin des années 2000, $90 \%$ avaient moins de cinq bovins (Hemme et Otte, 2010) mais produisaient $95 \%$ de la production laitière (Duteurtre et al., 2015a).

Cependant, la croissance de l'offre en lait, essentiellement basée sur la production laitière familiale, n'a pas permis de répondre à l'essor de la demande en produits laitiers (lait upérisé à haute température [UHT], yaourts) et les importations ont continué de croître. La production laitière nationale actuelle ne satisfait que $30 \%$ de la demande du pays (Nguyen Mai Huong et al., 2016) qui doit importer chaque année plus de 100000 tonnes de lait en poudre, notamment de NouvelleZélande, des Etats-Unis, des Pays-Bas, de France et d'Allemagne (Agroinfo, 2014). Afin de réduire le poids des importations en produits laitiers, les politiques publiques nationales soutiennent depuis 2008 le développement des exploitations laitières de grande taille, qu'elles soient familiales (plus de 30 vaches) ou industrielles (plus de 500 vaches). Les nouvelles politiques laitières visent aussi à favoriser l'intensification et la spécialisation laitière des exploitations, alors que celles-ci reposent traditionnellement sur une combinaison d'activités agricoles et/ou non-agricoles (Suzuki et al., 2006). Ainsi se développent au Vietnam de grandes, voire de très grandes exploitations, mises sur pied avec des capitaux privés, souvent à partir d'anciennes fermes d'Etat, et bénéficiant d'appuis des politiques publiques. Depuis 2005, plus d'une dizaine de fermes de plusieurs milliers de têtes ont été mises sur pied, avec comme objectif affiché de réduire la part des importations. En 2016, ces immenses fermes représentaient déjà $21 \%$ du cheptel laitier national, soit un effectif d'environ 53000 vaches laitières (Duteurtre et al., 2015b ; Nguyen Mai Huong et al., 2016).

Les exploitations familiales laitières continuent cependant à jouer un rôle très important pour le développement du secteur laitier au Vietnam (Duteurtre et al., 2015a). Dans ce contexte économique favorable à la production laitière, les modèles d'exploitations laitières familiales se diversifient, certaines exploitations s'agrandissent et se spécialisent alors que d'autres maintiennent une diversité d'activités agricoles et non-agricoles sur de petites surfaces.

Mais comment accompagner ce renouveau dans l'élevage pour qu'il soit durable, et quelles formes d'élevage privilégier? La durabilité de l'élevage est un enjeu pour le Vietnam comme pour de nombreux pays du Sud (Ness et al., 2007). Des études montrent par exemple que les grandes exploitations et les fermes spécialisées auraient des impacts négatifs pour l'environnement (pollution) et seraient plus vulnérables aux fluctuations des prix (Steinfeld et al., 2010). Les petites fermes pourraient être considérées comme plus durables sur le plan environnemental et social (Gura, 2008). Cependant, elles auraient également des contraintes telles que les charges de travail élevées ou encore les difficultés d'accès au marché, car elles restent peu équipées (tank à lait) (Gura, 2008 ; Hostiou et al., 2012).

Pour contribuer à la réflexion sur les modèles de fermes laitières à promouvoir, il est nécessaire de mieux connaître la diversité de ces exploitations laitières familiales et d'évaluer leur durabilité. Cet article a eu pour objectif de décrire la diversité et d'évaluer la durabilité sociale, environnementale et économique d'exploitations laitières familiales dans un district périurbain de Hanoï où la production laitière est en pleine croissance. La discussion porte sur les modèles d'exploitations laitières à appuyer ainsi que les mesures à mettre en place pour favoriser le développement de la production laitière.

\section{MATERIEL ET METHODES}

\section{Zone d'étude}

Au Vietnam, l'élevage laitier se développe essentiellement en zone périurbaine des grandes villes (Ho Chi Minh, Hanoï), comme dans le cas du district de $\mathrm{Ba}$ Vi qui se situe à 50 kilomètres de l'important marché de consommation de Hanoï (figure 1). Outre l'élevage laitier, le district de $\mathrm{Ba}$ Vi se caractérise par la diversité des activités agricoles (thé, patate douce, certains fruits et la poule de colline [gà ri]), touristiques (hôtels, etc.) ainsi que le développement des activités industrielles.

Le nombre de bovins laitiers et d'exploitations laitières ont fortement augmenté en passant de moins de 300 têtes en 1998 à plus de 7600 en 2014 (Duteurtre et al., 2015a), et de quelques dizaines d'exploitations en 1998 à 1407 en 2012. La production laitière est d'environ 20000 tonnes par an, soit 55000 litres par jour. Il existe une diversité de réseaux de collecte du lait à $\mathrm{Ba}$ Vi. La plupart des éleveurs vendent leur lait à des centres de collecte qui approvisionnent IDP, la principale laiterie industrielle. Les éleveurs et les centres de collectes sont liés par des contrats avec cette industrie laitière privée. Ce réseau concerne environ 1000 familles, soit près de $85 \%$ de la production de lait. Parmi celles-ci, les éleveurs localisés sur les terres de l'ancienne ferme d'Etat, actuellement Centre de recherche en élevage de Ba Vi (soit environ 200 familles), vendent leur lait à IDP par l'intermédiaire des centres de collectes du centre de recherche. Les autres centres de collecte sont privés.

Les enquêtes auprès d'éleveurs laitiers ont été réalisées dans les trois principales communes ayant développé la production laitière à $\mathrm{Ba} \mathrm{Vi}$ (Tan Linh, Van Hoa, Yen Bai), qui représentent plus de $70 \%$ de la production laitière totale du district (figure 2). Ces trois communes ont également été choisies par les politiques publiques pour investir et développer l'élevage laitier dans le programme d'aménagement de Hanoï. Elles présentent également une diversité en matière d'origine ethnique de la population (l'ethnie majoritaire Kinh, et l'ethnie Muong originaire de la zone), et en termes de formes d'accès au foncier : certaines exploitations possèdent des titres fonciers individuels, tandis que les exploitations laitières situées sur les terres du centre de recherche ne possèdent pas de titre foncier (Duteurtre et al., 2015a).

\section{Echantillon des exploitations enquêtées}

Au total 160 exploitations laitières dans ces trois communes ont été sélectionnées pour conduire les entretiens, soit $10 \%$ des exploitations laitières de la zone. Afin de couvrir la diversité des exploitations laitières sur ces trois communes, deux principaux critères de sélection ont été retenus : a) la taille du cheptel laitier (petite avec moins de 5 bovins laitiers, moyenne avec 5 à 10 bovins laitiers, grande avec plus de 10 bovins laitiers) car elle donne une indication sur les capacités de production des fermes, et b) la diversité des activités agricoles et non-agricoles dans les exploitations (exploitations spécialisées dans la production laitière, lait + activité agricole, lait + activité agricole + activité non-agricole). Une ferme laitière industrielle de grande dimension mise en place par IDP a également été enquêtée, même s'il s'agit d'une exception vis-à-vis des autres exploitations familiales. L'objectif était de connaître son fonctionnement ainsi que ses performances en terme de durabilité. Les données utilisées dans la typologie des exploitations ainsi que dans l'analyse de durabilité sont basées sur l'année 2013. 


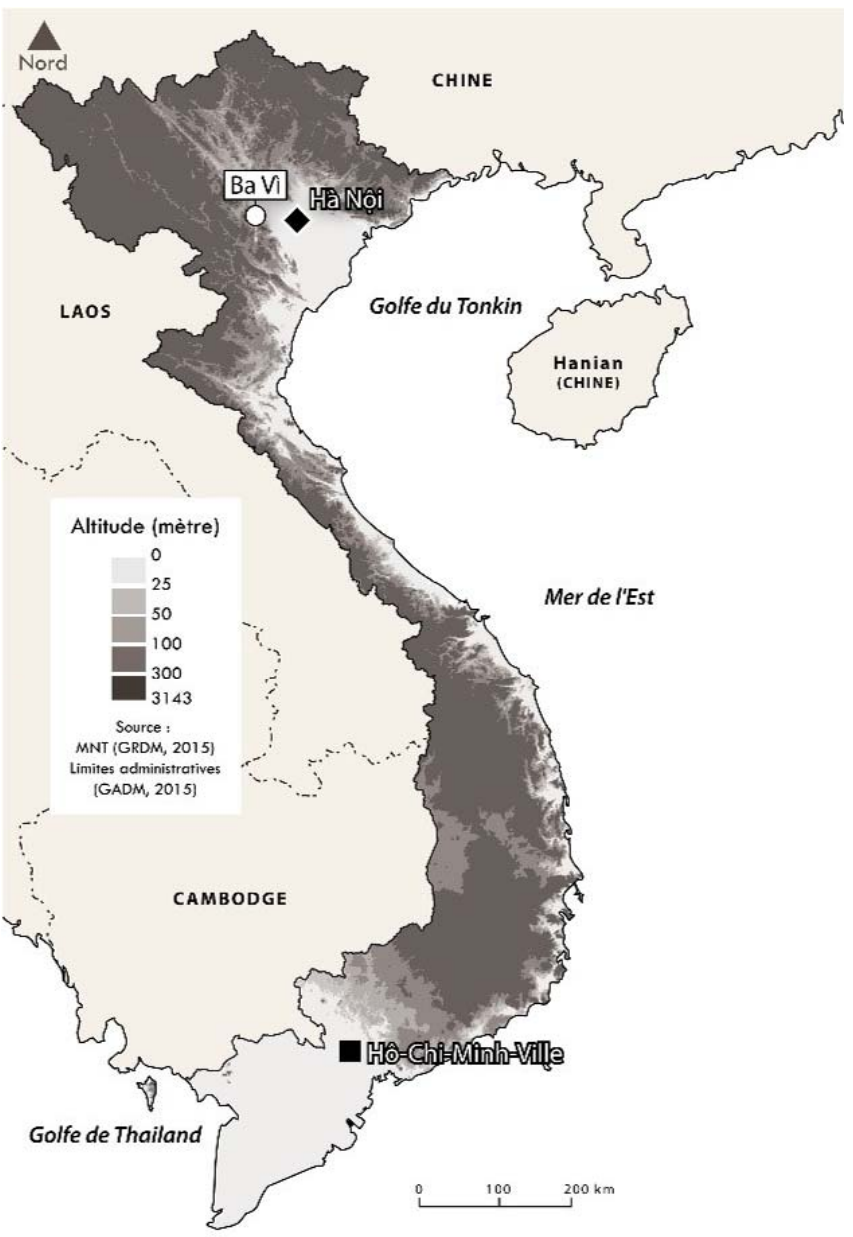

Figure 1 : localisation du district de Ba Vi au Vietnam. (Carte : J.-D. Cesaro)

\section{Recueil des données}

Les entretiens ont été réalisés à l'aide d'un questionnaire directif. Les questions ont porté sur les capitaux disponibles sur l'exploitation, parce que la diversité des exploitations agricoles se base sur les ressources et les objectifs des agriculteurs, en référence au cadre d'analyse des moyens d'existence durables (Carney et al., 1999 ; Bosc et al., 2014), sur le fonctionnement technique de l'élevage (pratiques d'alimentation, de reproduction, de traite, et de commercialisation), et sur les performances économiques, environnementales et sociales de l'exploitation. Les critères à renseigner pour chaque thème (capitaux, fonctionnement technique et performances) ont été choisis en fonction d'une synthèse de la littérature sur la durabilité des systèmes d'élevage (Vilain et al., 2008 ; Lebacq et al., 2013 ; Chand et al., 2015 ; Salas-Reyes et al., 2015) et des discussions avec les experts locaux (Lopez-Ridaura et al., 2002). Comme peu d'exploitants disposaient de comptes bancaires pour la gestion de leur trésorerie, nous avons choisi le revenu brut (ou produit brut d'exploitation) comme indicateur du capital financier car il rend compte du volume de trésorerie disponible sur l'exploitation. Toutes les données sont présentées en dollars US ( 1 \$ = 21000 dôngs vietnamiens au moment de l'enquête).

\section{Analyse des données}

Afin de décrire la diversité des exploitations familiales, une analyse des données recueillies lors des entretiens a été réalisée en plusieurs étapes. Dans la première, des variables ont été construites pour décrire les exploitations d'après leur dotation en capitaux (Chambers et al., 1992) et le fonctionnement de l'atelier laitier (tableau I). Des variables

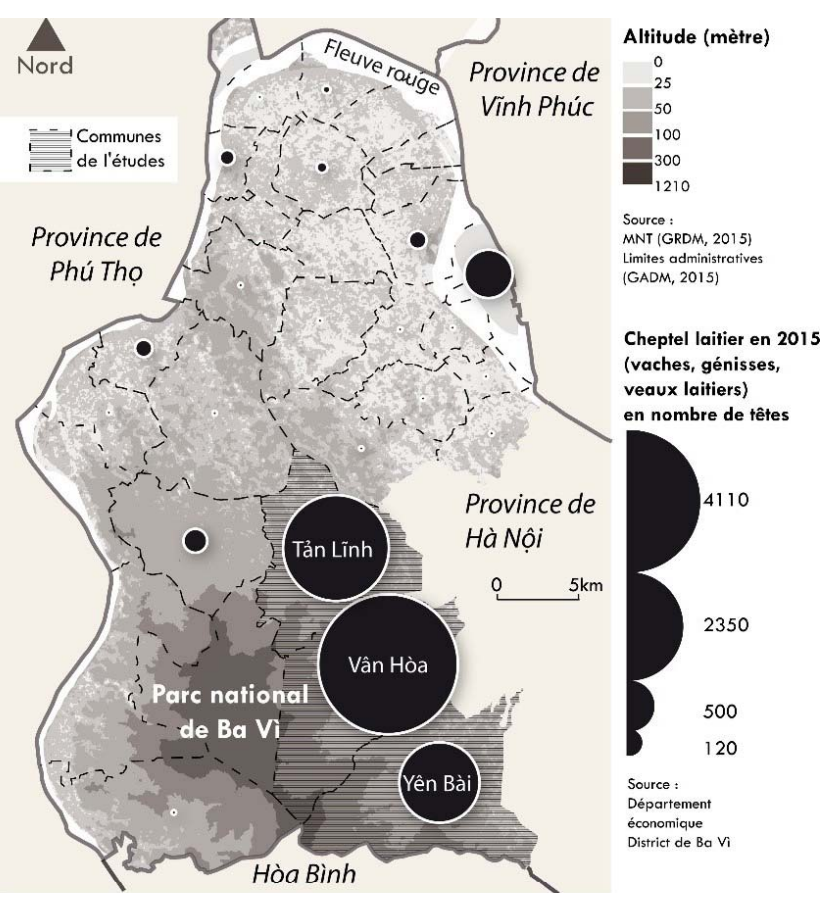

Figure 2 : localisation du cheptel laitier dans le district de Ba Vi au Vietnam en 2015. (Carte : J.-D. Cesaro)

\section{Tableau I}

Variables utilisées pour décrire les dotations en capitaux et le fonctionnement technique des exploitations laitières enquêtées dans le district de Ba Vi au Vietnam (2013)

\begin{tabular}{|c|c|}
\hline $\begin{array}{l}\text { Thèmes } \\
\text { (nb. variables) }\end{array}$ & Variables actives \\
\hline Capital naturel (2) & $\begin{array}{l}\text { Surface totale (ha) } \\
\text { Surface en fourrages/SAU }{ }^{1}(\%)\end{array}$ \\
\hline Capital physique (3) & $\begin{array}{l}\text { Machine à traire (oui/non) } \\
\text { Générateur électrique (oui/non) } \\
\text { Nb. bovins laitiers (vaches et génisses) }\end{array}$ \\
\hline Capital humain (6) & $\begin{array}{l}\text { Ancienneté de la production laitière (ans) } \\
\text { Groupe ethnique (Kinh/Muong) } \\
\text { Nb. personnes dans la famille (personnes) } \\
\text { Nb. travailleurs sur l'élevage laitier (travailleurs) } \\
\text { Heures de travail réalisées par les femmes (\%) } \\
\text { Niveau d'éducation du chef d'exploitation } \\
\text { (primaire/collège/lycée/université) }\end{array}$ \\
\hline Capital social (2) & $\begin{array}{l}\text { Type de débouché pour la vente du lait } \\
\text { et relation avec l'acheteur } \\
\text { Activités non-agricoles (oui/non) }\end{array}$ \\
\hline Capital financier (3) & $\begin{array}{l}\text { Revenu total issu d'activités agricoles } \\
\text { et non-agricoles = revenu brut = produit } \\
\text { brut d'exploitation }(\$ / \text { an) } \\
\text { Revenu total issu d'activités agricoles } \\
\text { et non-agricoles / travailleur familial (\$/an) } \\
\text { Revenu des activités agricoles par SAU (\$/ha) }\end{array}$ \\
\hline $\begin{array}{l}\text { Fonctionnement de } \\
\text { l'atelier laitier (1) }\end{array}$ & Autonomie fourragère (oui/non) \\
\hline
\end{tabular}

${ }^{1}$ Surface agricole utile 
supplémentaires ont également été utilisées dans l'analyse : production laitière (L/an) et surface fourragère (ha / vache laitière). L'évaluation de la durabilité des exploitations a reposé sur l'analyse des trois facettes de la durabilité (économique, sociale, environnementale) pour l'atelier laitier (tableau II). Les variables de durabilité ont été sélectionnées à partir de la bibliographie et de réunions entre experts.

Pour l'analyse de la durabilité économique de l'atelier laitier, nous avons mobilisé trois variables décrivant la marge brute par exploitation, la marge brute par surface fourragère et la marge brute par travailleur (tableau II), en nous appuyant sur la bibliographie (Brossier et al., 2003) et sur des réunions entre experts. La marge brute correspond à la somme des revenus laitiers, desquels ont été soustraites les charges opérationnelles (hors travail familial). Les revenus laitiers incluent les ventes de lait, d'animaux et de fumier. Les charges opérationnelles (hors travail familial) incluent le coût des aliments, des produits et services vétérinaires, et du travail salarié. Elles n'incluent pas les charges de structure (accès à la terre, bâtiments, achats d'animaux, de machine à traire, de biogaz). Deux autres variables ont été retenues : nombre d'activités générant des revenus sur l'exploitation et part du revenu du lait sur le revenu total de l'exploitation. Ces variables permettent de rendre compte du niveau de diversification des activités et donc des capacités d'adaptation des ménages à des variations du prix du lait qui ont été marquantes dans le district de $\mathrm{Ba}$ Vi ces dernières années (tableau II).

Pour la dimension sociale de la durabilité, trois variables rendant compte du temps de travail dans l'atelier laitier (tableau II) ont été retenues car les charges de travail sont une contrainte au développement durable de la production laitière dans ces exploitations (Hostiou et al., 2012). Pour la durabilité environnementale, deux variables (tableau II) ont été mobilisées pour mesurer l'impact des cultures fourragères sur la pollution des sols et de l'eau du fait de l'utilisation d'herbicides et d'engrais par les éleveurs.

Dans une deuxième étape, une analyse multivariée a été réalisée pour construire une typologie d'exploitations. Elle a consisté en une analyse factorielle multiple (AFM ; Escofier et Pagès, 1994) sur les six groupes de variables actives décrivant les capitaux et le fonctionnement de l'atelier lait (tableau I), suivie d'une classification ascendante hiérarchique $(\mathrm{CAH})$. La CAH a été réalisée à partir des principaux facteurs issus de l'AFM sur les critères regroupés par thème décrivant les exploitations laitières (tableau I). L'AFM permet, grâce à une pondération propre à chaque groupe de variables, de mieux rendre compte de leur hétérogénéité dans la description de la diversité des exploitations. L'analyse statistique a été réalisée avec ade4 (Dray et al., 2007) et le logiciel de statistique R (R Core Team, 2016).

Ensuite, une représentation avec un graphe radar a été retenue pour représenter et comparer les profils de durabilité des différents types d'exploitations laitières (Lairez et al., 2015). Nous avons utilisé une échelle linéaire de 10 points pour représenter la valeur de chaque variable. La valeur des variables a varié de l'indice 1 (minimum dans l'échantillon) à l'indice 10 (maximum). L'exploitation de grande taille (appartenant à la laiterie privée IDP) a été analysée à part : ses caractéristiques ont été présentées en lien avec la description des types et de la durabilité réalisée pour les exploitations familiales de l'échantillon.

\section{RESULTATS}

\section{Présentation de l'échantillon}

Les caractéristiques générales des 160 exploitations laitières enquêtées à $\mathrm{Ba}$ Vi sont présentées dans le tableau III. En moyenne les exploitations ont une surface de 0,84 hectare et possèdent 3,4 vaches pour une production laitière annuelle de 13600 litres. La production laitière est une activité relativement récente ayant débuté en moyenne

\section{Tableau III}

Caractéristiques générales des exploitations laitières $(n=160)$ enquêtées dans le district de Ba Vi au Vietnam (2013)

\begin{tabular}{|c|c|c|c|}
\hline Variables & Minimum & Moyenne & Maximum \\
\hline Surface totale (ha) & 0,24 & 0,84 & 6 \\
\hline $\begin{array}{l}\text { Nb. bovins laitiers } \\
\text { (vaches et génisses) }\end{array}$ & 1 & 6 & 25 \\
\hline $\mathrm{Nb}$. vaches laitières (traites et taries) & es) 1 & 3,4 & 16 \\
\hline $\begin{array}{l}\text { Début de la production laitière } \\
\text { (année) }\end{array}$ & 1986 & 2004 & 2013 \\
\hline Nb. personnes dans la famille & 2 & 4 & 13 \\
\hline $\begin{array}{l}\text { Main d'œuvre familiale } \\
\text { (nb. travailleurs sur la ferme) }\end{array}$ & 1 & 2,4 & 5 \\
\hline $\begin{array}{l}\text { Production laitière totale } \\
\text { vendue }(\mathrm{L})\end{array}$ & 3000 & 13600 & 55000 \\
\hline
\end{tabular}

\section{Tableau II}

Variables de performances utilisées pour analyser la durabilité de l'atelier laitier des exploitations laitières enquêtées dans le district de Ba Vi au Vietnam (2013)

\begin{tabular}{|c|c|}
\hline Thèmes (nb. variables) & Variables \\
\hline $\begin{array}{l}\text { Performances } \\
\text { économiques (5) }\end{array}$ & $\begin{array}{l}\text { Marge brute totale de l'atelier laitier par exploitation par an (\$/exploitation/an) } \\
\text { Marge brute totale de l'atelier laitier par travailleur familial sur l'atelier laitier et par an (\$/travailleur/an) } \\
\text { Marge brute totale de l'atelier laitier par ha de surface fourragère par an (\$/ha/an) } \\
\text { Nb. activités générant des revenus sur l'exploitation } \\
\text { Part du revenu du lait sur le revenu total de l'exploitation }(\%)\end{array}$ \\
\hline $\begin{array}{l}\text { Performances } \\
\text { environnementales (2) }\end{array}$ & $\begin{array}{l}\text { Montant total des achats } d^{\prime} \text { herbicides pour la surface fourragère }(\$ / \text { ha/an) } \\
\text { Montant total des achats d'engrais (NPK) }{ }^{1} \text { pour la surface fourragère }(\$ / \mathrm{ha} / \mathrm{an})\end{array}$ \\
\hline Performances sociales (3) & $\begin{array}{l}\text { Temps de travail total de l'atelier laitier par exploitation et par an (h/exploitation/an) } \\
\text { Temps de travail total de l'atelier laitier par travailleur sur l'atelier laitier et par an (h/travailleur/an) } \\
\text { Temps de travail total de l'atelier laitier par bovin laitier et par an (h / bovin laitier / an) }\end{array}$ \\
\hline
\end{tabular}

${ }^{1}$ Azote, phosphore, potassium 
en 2004. La main d'œuvre comprend en moyenne 2,4 travailleurs familiaux par ferme. Ces moyennes cachent évidemment d'importantes disparités dans les dotations en capitaux et dans les performances de production.

Au moment de l'enquête, les pratiques de conduite du troupeau laitier présentaient un certain nombre de points communs entre les fermes. Elles reposaient sur un modèle technique intensif en travail et en terre, avec une distribution à l'auge de fourrages cultivés sur l'exploitation (herbe à éléphant [Pennisetum purpureum] et maïs) et de concentrés achetés, l'élevage de vaches métisses toutes issues de croisement avec la race Holstein, et la pratique généralisée de l'insémination artificielle. La diversité des pratiques d'élevage observées reposait surtout sur l'autonomie fourragère et sur les modes de commercialisation du lait, variables retenues pour la typologie (tableau I). Les variables décrivant les pratiques d'élevage n'ont pas été retenues comme discriminantes dans la typologie, celle-ci étant construite sur les cinq capitaux.

\section{Caractérisation de la diversité des exploitations laitières}

La typologie a permis d'identifier cinq types d'exploitations laitières (tableaux IV et V ; encadré 1) dont trois étaient des exploitations avec une surface agricole moyenne (environ un hectare), et deux avaient une petite surface agricole (environ un demi-hectare). Parmi les exploitations avec une surface agricole moyenne les trois types décrits ci-après ont été distingués (tableau IV).

Les exploitations du type 1 associent une diversité d'activités agricoles (riz, porcs, volailles) et non-agricoles (entre autres commerce, emploi), avec plus de six activités en moyenne. La part des terres dédiées à la culture des fourrages pour alimenter les vaches laitières est la plus faible de l'échantillon (70 \%), laissant ainsi une place relativement importante aux cultures vivrières. La production de lait est récente et peu développée : le cheptel est de petite taille (3,8 bovins laitiers), il n'y a pas d'équipement (ni machine à traire ni générateur électrique) et le volume produit est l'un des plus faibles de l'échantillon. Ces exploitations sont autonomes en fourrages pour alimenter les vaches toute l'année avec une surface fourragère par vache laitière la plus élevée de l'échantillon. Le lait est vendu exclusivement à l'industrie laitière privée de la province (IDP). Dans ces familles d'origine Muong et de grande taille, le nombre de personnes impliquées dans l'activité laitière est le plus grand de l'échantillon $(2,9)$, avec une contribution importante des femmes.

Les exploitations du type 2 (encadré 1) reposent sur plusieurs activités agricoles (4,3 en moyenne) dont le lait, d'autres élevages (porcs) et des cultures comme le riz, le manioc, le maïs. En revanche, elles n'ont pas d'activités non-agricoles. L'élevage laitier comprend un troupeau de grande taille ( 8,2 têtes) et un atelier bien équipé (générateur

Encadré 1

Type 1 : exploitations mixtes pluriactives peu intensives avec un petit troupeau laitier

Type 2 : exploitations de polyculture-élevage intensives, avec un troupeau laitier relativement grand

Type 3 : exploitations de polyculture-élevage moyennement intensives avec un troupeau laitier de taille moyenne

Type 4 : petites exploitations pluriactives peu intensives avec un petit troupeau laitier

Type 5 : exploitations spécialisées en lait et intensives, avec un troupeau laitier relativement grand électrique, machine à traire). La quasi-totalité de la surface est cultivée en fourrages. Cependant, ces exploitations ne sont pas autonomes en fourrages en hiver : à cette saison, les éleveurs doivent acheter divers types de fourrages et de sous-produits agricoles, ainsi que du maïs ensilé produit localement pour faire de l'ensilage. Les éleveurs sont d'origine Kinh. En moyenne, deux personnes travaillent sur l'atelier laitier, avec une faible contribution des femmes.

Les exploitations du type 3 (encadré 1) se caractérisent par une diversité encore plus importante des activités agricoles élevage et cultures (5,7 en moyenne). Le troupeau laitier est de taille moyenne par rapport à l'échantillon (5,7 bovins par exploitation) et $80 \%$ de la surface est cultivée en fourrages. La plupart des exploitants sont d'origine Muong. Le nombre de travailleurs impliqués dans l'atelier laitier est élevé par rapport à l'échantillon (2,6 en moyenne). Les éleveurs vendent exclusivement leur lait à IDP.

Parmi les exploitations avec une petite surface agricole (environ un demi-hectare), on distingue deux types (types 4 et 5 ; tableau IV ; encadré 1). Les exploitations du type 4 sont très proches du type 2 mais elles ont deux fois moins de terres et d'animaux laitiers (3,9 en moyenne) et sont moins bien équipées. Elles sont pour la plupart localisées sur le centre de recherche (figure 3). Les ménages sont investis dans un grand nombre d'activités agricoles et non-agricoles (5,1 en moyenne). Une seule personne s'occupe de l'élevage laitier, sans machine à traire ni générateur. Ces exploitations ont recours à plusieurs modes de commercialisation du lait (boutiques et centre de recherche de $\mathrm{Ba} \mathrm{Vi})$. Ces éleveurs sont d'origine Kinh et ont un niveau d'éducation élevé par rapport à l'échantillon.

Dans les exploitations du type 5 il n'y a pas d'activité non-agricole et peu de diversité des activités agricoles. Le cheptel est de grande taille avec en moyenne 7,3 bovins laitiers. La surface fourragère par vache est la plus faible de l'échantillon ( $0,19 \mathrm{ha} /$ vache) et les exploitations ne sont pas autonomes en fourrages pour alimenter les vaches toute l'année. Elles doivent donc recourir à des achats d'aliments. Ces exploitations se caractérisent par des ménages de petite taille par rapport aux autres groupes (3,8 personnes par famille en moyenne). Deux personnes s'occupent de la production laitière en bénéficiant d'équipements (machine à traire, générateur). Ces éleveurs sont d'origine Kinh. Ils sont tous installés sur le centre de recherche et bénéficient à ce titre de l'appui technique qu'il fournit (figure 3). Ils ont recours à plusieurs modes de commercialisation du lait (boutiques et centre de recherche de $\mathrm{Ba} \mathrm{Vi}$ ).

Enfin, le dernier type d'exploitation est composé d'une seule ferme présente sur le district de $\mathrm{Ba} \mathrm{Vi}$ : la ferme laitière industrielle mise en place par la laiterie privée IDP. Cette exploitation est spécialisée dans la production de lait et de génisses. Elle est de très grande dimension

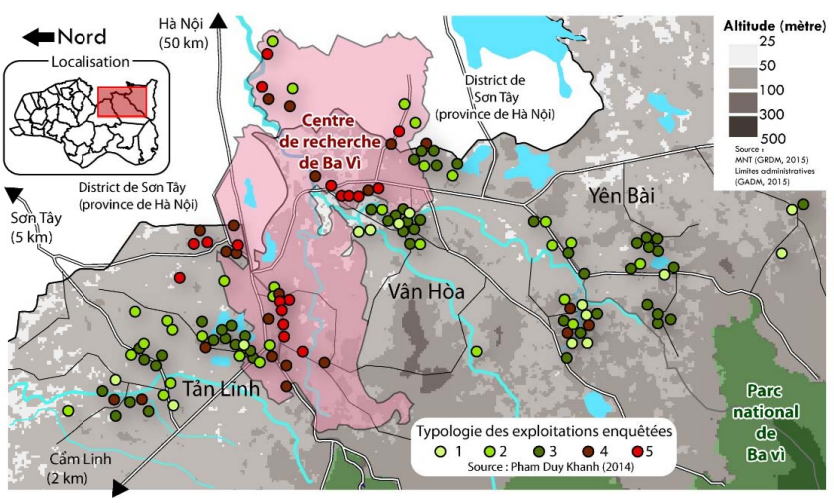

Figure 3 : localisation des cinq types d'exploitations laitières enquêtées dans le district de Ba Vi au Vietnam. (Carte : J.-D. Cesaro) 


\section{Tableau IV}

Caractéristiques des cinq types d'exploitations (expl) laitières et de la ferme industrielle enquêtées dans le district de Ba Vi au Vietnam (2013) (moyenne \pm écart-type)

\begin{tabular}{|c|c|c|c|c|c|c|c|}
\hline \multirow[t]{3}{*}{ 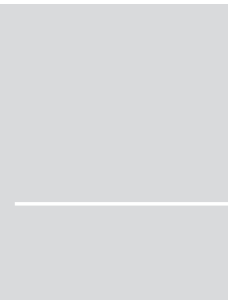 } & \multirow[t]{2}{*}{ Variables } & Type 1 & Type 2 & Type 3 & Type 4 & Type 5 & \multirow{2}{*}{$\begin{array}{c}\begin{array}{c}\text { Ferme } \\
\text { industrielle }\end{array} \\
\begin{array}{l}\text { Très grande } \\
\text { exploitation }\end{array}\end{array}$} \\
\hline & & \multicolumn{3}{|c|}{$\begin{array}{l}\text { Exploitation avec une surface } \\
\text { agricole moyenne }(\approx 1 \text { ha })\end{array}$} & \multicolumn{2}{|c|}{$\begin{array}{l}\text { Exploitation avec une petite } \\
\text { surface agricole }(\approx 1 / 2 \mathrm{ha})\end{array}$} & \\
\hline & $\begin{array}{l}\text { Pourcentage de } \\
\text { l'échantillon }\end{array}$ & $\begin{array}{c}10 \% \\
(15 \text { expl) }\end{array}$ & $\begin{array}{c}22 \% \\
(35 \text { expl) }\end{array}$ & $\begin{array}{c}33 \% \\
(53 \text { expl) }\end{array}$ & $\begin{array}{c}19 \% \\
(31 \text { expl) }\end{array}$ & $\begin{array}{c}16 \% \\
(25 \text { expl) }\end{array}$ & 1 expl \\
\hline \multirow[t]{2}{*}{ Capital naturel } & Surface totale (ha) & $0,9 \pm 0,3$ & $1 \pm 0,45$ & $0,9 \pm 0,42$ & $0,5 \pm 0,25$ & $0,6 \pm 0,30$ & 15 \\
\hline & $\begin{array}{l}\text { Pourcentage de } \\
\text { la surface en } \\
\text { fourrages / SAU }{ }^{1}\end{array}$ & $70 \%$ & $90 \%$ & $80 \%$ & $70 \%$ & $80 \%$ & $60 \%$ \\
\hline \multirow[t]{4}{*}{ Capital physique } & Machine à traire & Non & Oui & $+/-$ & Non & Oui & Oui \\
\hline & Générateur électrique & Non & Oui & $+/-$ & Non & Oui & Oui \\
\hline & $\begin{array}{l}\text { Nb. bovins laitiers } \\
\text { (vaches et génisses) }\end{array}$ & $3,8 \pm 1,5$ & $8,2 \pm 4,0$ & $5,7 \pm 2,0$ & $3,9 \pm 1,6$ & $7,3 \pm 3,8$ & 184 \\
\hline & $\begin{array}{l}\text { Dont vaches laitières } \\
\text { adultes }\end{array}$ & 1,9 & 4,7 & 3,3 & 2,2 & 4,3 & 96 \\
\hline \multirow[t]{6}{*}{ Capital humain } & $\begin{array}{l}\text { Ancienneté de la } \\
\text { production laitière }\end{array}$ & $6,5 \pm 4,6$ & $9,2 \pm 5,2$ & $7,5 \pm 4,4$ & $8,2 \pm 5,2$ & $14,6 \pm 5,6$ & 2012 \\
\hline & Groupe ethnique & Muong & Kinh & Muong & $+/-$ & Kinh & Kinh \\
\hline & $\begin{array}{l}\text { Nb. personnes dans } \\
\text { la famille }\end{array}$ & $5,5 \pm 1,6$ & $4,4 \pm 1,0$ & $4,8 \pm 1,2$ & $4,0 \pm 1,1$ & $3,8 \pm 1,1$ & 0 \\
\hline & $\begin{array}{l}\text { Nb. travailleurs sur } \\
\text { l'élevage laitier }\end{array}$ & $2,9 \pm 0,7$ & $2,2 \pm 0,6$ & $2,6 \pm 0,8$ & $1,7 \pm 0,8$ & $2,1 \pm 0,5$ & 14 \\
\hline & $\begin{array}{l}\text { Pourcentage des heures } \\
\text { de travail réalisées par } \\
\text { les femmes }\end{array}$ & $>50 \%$ & $<50 \%$ & $+/-$ & $+/-$ & $+/-$ & l \\
\hline & Niveau d'éducation & $+/-$ & $+/-$ & $+/-$ & Lycée & $+/-$ & l \\
\hline \multirow[t]{2}{*}{ Capital social } & $\begin{array}{l}\text { Type de débouché } \\
\text { pour la vente du lait et } \\
\text { relation avec l'acheteur }\end{array}$ & Laiterie privée & $+/-$ & Laiterie privée & $\begin{array}{l}\text { Centre de } \\
\text { recherche } \\
+ \text { boutiques } \\
\text { privées }\end{array}$ & $\begin{array}{l}\text { Centre de } \\
\text { recherche } \\
+ \text { boutiques } \\
\text { privées }\end{array}$ & Laiterie privée \\
\hline & Activités non-agricoles & Oui & Non & $+/-$ & Oui & Non & Non \\
\hline \multirow[t]{3}{*}{ Capital financier } & $\begin{array}{l}\text { Revenu brut total issu } \\
\text { d'activités agricoles et } \\
\text { non-agricoles (\$/an) }\end{array}$ & $\begin{array}{r}11500 \\
\pm 4950\end{array}$ & $\begin{array}{r}17000 \\
\pm 6900\end{array}$ & $\begin{array}{r}16400 \\
\pm 9380\end{array}$ & $9500 \pm 4420$ & $\begin{array}{r}13550 \\
\pm 6350\end{array}$ & 210000 \\
\hline & $\begin{array}{l}\text { Revenu des activités } \\
\text { agricoles par SAU (\$/ha) }\end{array}$ & $\begin{array}{r}12500 \\
\pm 8800\end{array}$ & $\begin{array}{r}19500 \\
\pm 9150\end{array}$ & $\begin{array}{l}18500 \\
\pm 12100\end{array}$ & $\begin{array}{l}21500 \\
\pm 17850\end{array}$ & $\begin{array}{l}23000 \\
\pm 12800\end{array}$ & 14000 \\
\hline & $\begin{array}{l}\text { Revenu brut total de } \\
\text { toutes activités (agricoles } \\
+ \text { non-agricoles) / } \\
\text { travailleur familial (\$/an) }\end{array}$ & $\begin{array}{r}3150 \\
\pm 2660\end{array}$ & $\begin{array}{l}5800 \\
\pm 3060\end{array}$ & $\begin{array}{r}5150 \\
\pm 3255\end{array}$ & $\begin{array}{r}3400 \\
\pm 1595\end{array}$ & $\begin{array}{r}4850 \\
\pm 2145\end{array}$ & $\begin{array}{l}15000 \$ \text { / } \\
\text { travailleur } \\
(100 \% \\
\text { salariés })\end{array}$ \\
\hline $\begin{array}{l}\text { Fonctionnement } \\
\text { de l'atelier laitier }\end{array}$ & Autonomie fourragère & Oui & Non & Oui & Non & Non & non \\
\hline \multirow[t]{3}{*}{$\begin{array}{l}\text { Variables } \\
\text { supplémentaires }\end{array}$} & $\begin{array}{l}\text { Production laitière } \\
\text { (L lait / an) }\end{array}$ & $\begin{aligned} & 8733 \\
\pm & 4697,5\end{aligned}$ & $\begin{array}{c}18229 \\
\pm 5956,1\end{array}$ & $\begin{array}{l}12774 \\
\pm 5272,0\end{array}$ & $\begin{aligned} & 8645 \\
\pm & 3834,5\end{aligned}$ & $\begin{array}{l}16160 \\
\pm 8168,4\end{array}$ & 300000 \\
\hline & $\begin{array}{l}\text { Surface fourragère / } \\
\text { vache laitière (ha/vache) }\end{array}$ & $0,41 \pm 0,21$ & $0,29 \pm 0,1$ & $0,24 \pm 0,12$ & $0,21 \pm 0,12$ & $0,19 \pm 0,11$ & 0,16 \\
\hline & $\begin{array}{l}\mathrm{Nb} \text {. litres de lait / ha } \\
\text { de surface fourragère }\end{array}$ & $\begin{array}{l}17000 \\
\pm 18000\end{array}$ & $\begin{array}{r}24000 \\
\pm 11000\end{array}$ & $\begin{array}{r}19000 \\
\pm 9000\end{array}$ & $\begin{array}{r}28000 \\
\pm 24000\end{array}$ & $\begin{array}{l}28000 \\
\pm 14000\end{array}$ & 20000 \\
\hline
\end{tabular}


par rapport aux autres exploitations de l'échantillon, avec une surface de 15 hectares en fourrages et 184 bovins laitiers Holstein (dont 96 vaches laitières) pour une production annuelle de 300 tonnes de lait vendu en totalité à la laiterie IDP. L'exploitation est équipée de tracteurs, d'une salle de traite et d'un distributeur de concentré. La main d'œuvre salariée se compose de 12 salariés permanents, d'un vétérinaire, d'une comptable et d'un directeur de la ferme. Créée en 2012, elle est l'une des fermes les plus récentes de l'échantillon.

\section{Durabilité des exploitations laitières}

Cette première approche de l'évaluation de la durabilité des exploitations résulte de l'analyse conjointe des performances économiques, environnementales et sociales. Avant d'étudier ces trois dimensions de manière intégrée, les performances des fermes pour chacune de ces dimensions ont été examinées (figure 4 ; tableau V).

Les performances économiques des exploitations doivent d'abord être abordées au niveau de l'atelier laitier. La marge brute de l'atelier laitier est plus élevée dans les exploitations les plus intensives (types 2 et 5) puisque celles-ci possèdent un troupeau laitier plus important et un meilleur niveau d'équipement. Elles dégagent une marge brute par exploitation comprise en moyenne entre 6400 et 7600 \$an. Les fermes les plus intensives et les mieux équipées (types 2 et 5) dégagent également les marges brutes par travailleur et par unité de surface

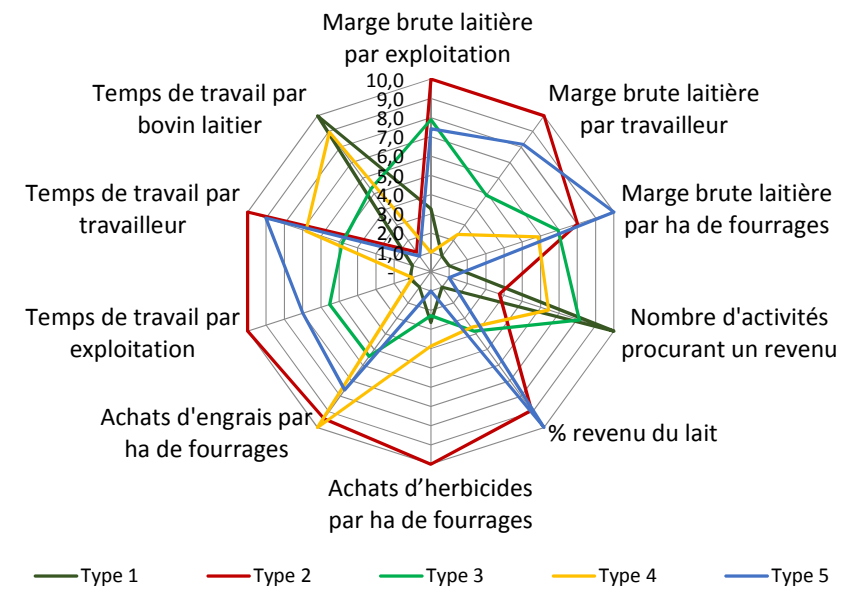

Figure 4 : résultats d'analyse des performances économique, environnementale et sociale des exploitations laitières du district de Ba Vi au Vietnam en 2013.

fourragère les plus élevées. Pour ces deux types, les marges brutes par travailleur sont en moyenne de 3500 à $4000 \$$ /travailleur/an, et les marges brutes par hectare sont de l'ordre de 9200 à 10000 \$/ha/ an. Il est intéressant de noter que parmi ces deux types d'exploitations

\section{Tableau V}

Résultats de durabilité des exploitations laitières enquêtées dans le district de Ba Vi au Vietnam selon les variables associées aux trois types de performances (2013)

\begin{tabular}{|c|c|c|c|c|c|c|c|}
\hline $\begin{array}{l}\text { Thèmes } \\
\text { (nb. variables) }\end{array}$ & Variables & Type 1 & Type 2 & Type 3 & Type 4 & Type 5 & $\begin{array}{c}\text { Ferme } \\
\text { industrielle }\end{array}$ \\
\hline \multirow[t]{6}{*}{$\begin{array}{l}\text { Performances } \\
\text { économiques (5) }\end{array}$} & $\begin{array}{l}\text { Marge brute totale de l'atelier laitier } \\
\text { par exploitation et par an }(\$)\end{array}$ & 4452 & 7559 & 6587 & 3413 & 6370 & 76710 \\
\hline & \multirow[t]{2}{*}{$\begin{array}{l}\text { Marge brute totale de l'atelier laitier } \\
\text { par travailleur familial sur l'atelier laitier } \\
\text { et par an (\$/travailleur/an) }\end{array}$} & \multirow[t]{2}{*}{1347} & \multirow[t]{2}{*}{3939} & \multirow[t]{2}{*}{2474} & \multirow[t]{2}{*}{1748} & \multirow[t]{2}{*}{3410} & $\begin{array}{l}\text { Gérant : une part des } \\
76710 \text { \$/an } \\
\text { Salaires des ouvriers : } \\
1800\end{array}$ \\
\hline & & & & & & & $\begin{array}{l}\text { Salaires des employés } \\
\text { qualifiés : } 3600\end{array}$ \\
\hline & $\begin{array}{l}\text { Marge brute totale de l'atelier laitier par } \\
\text { ha de surface fourragère par an (\$/ha/an) }\end{array}$ & 6228 & 9153 & 8710 & 8258 & 9980 & 5114 \\
\hline & Nombre de revenus de l'exploitation & 3,5 & 2,2 & 3,1 & 2,8 & 1,6 & 1,0 \\
\hline & $\begin{array}{l}\text { Part du revenu du lait sur le revenu brut } \\
\text { total de l'exploitation (\%) }\end{array}$ & 55,0 & 85,8 & 66,0 & 65,0 & 90,0 & 100,0 \\
\hline \multirow[t]{2}{*}{$\begin{array}{l}\text { Performances envi- } \\
\text { ronnementales ( } 2 \text { ) }\end{array}$} & $\begin{array}{l}\text { Montant total des achats } \mathrm{d}^{\prime} \text { herbicides pour } \\
\text { la surface fourragère }(\$ / \mathrm{ha} / \mathrm{an})\end{array}$ & 32,5 & 48,5 & 31,8 & 35,2 & 29,0 & 70,0 \\
\hline & $\begin{array}{l}\text { Montant total des achats d'engrais (NPK) }{ }^{1} \\
\text { pour la surface fourragère (\$/ha/an) }\end{array}$ & 121,5 & 200,5 & 163,1 & 205,7 & 183,2 & 300,0 \\
\hline \multirow[t]{3}{*}{$\begin{array}{l}\text { Performances } \\
\text { sociales ( } 3 \text { ) }\end{array}$} & $\begin{array}{l}\text { Temps de travail total de l'atelier laitier par } \\
\text { exploitation et par an (h/exploitation/an) }\end{array}$ & 2239 & 3462 & 2844 & 2219 & 3046 & 31200 \\
\hline & $\begin{array}{l}\text { Temps de travail total de l'atelier laitier } \\
\text { par travailleur sur l'atelier laitier et par an } \\
\text { (h/travailleur/an) }\end{array}$ & 763 & 1554 & 1100 & 1274 & 1467 & 2400 \\
\hline & $\begin{array}{l}\text { Temps de travail total de l'atelier laitier par } \\
\text { bovin laitier et par an (h / bovin laitier / an) }\end{array}$ & 589 & 422 & 499 & 569 & 417 & 169 \\
\hline
\end{tabular}

${ }^{1}$ Azote, phosphore, potassium 
les plus rentables, on trouve des exploitations de type 2 ainsi que des exploitations spécialisées (type 5). Cependant, les exploitations de polyculture-élevage de type 2, à l'instar des exploitations mixtes et/ ou pluriactives moins intensives (types 1, 3 et 4), sont moins vulnérables économiquement à des aléas sur les prix du lait en raison de leur capacité à compenser des baisses de revenu laitier par d'autres activités agricoles et/ou non-agricoles. Au contraire, la spécialisation des exploitations les plus intensives (type 2) peut rendre ces exploitations plus fragiles face à des variations du prix du lait. La part de la production laitière dans le revenu global des exploitations les moins intensives est en effet inférieure à $65 \%$ (tableau $\mathrm{V}$; figure 4 ).

La grande ferme industrielle (IDP) se différencie fortement des autres fermes par ses performances économiques. En raison de la taille de son cheptel et de l'importance de ses équipements, elle dégage une marge brute laitière environ 10 à 20 fois supérieure à celle des exploitations familiales. Par ailleurs, la productivité du travail de la ferme IDP est très supérieure à celle des autres fermes : elle possède 13 vaches par travailleur alors que les fermes familiales élèvent en moyenne 2,3 vaches par travailleur (tableau III). La productivité de la grande ferme est de 21428 litres par travailleur et par an, alors qu'elle est de 5666 litres par travailleur dans les petites exploitations. Cette productivité du travail se traduit sur la marge brute par travailleur. Pour les ouvriers qui perçoivent un salaire de 1800 \$/an, le revenu par travailleur est comparable à la marge brute laitière des fermes les moins intensives. Pour les employés qualifiés (vétérinaire et comptable) qui sont payés 3600 \$/an, le salaire est équivalent à la marge brute par travailleur des fermes les plus intensives. Mais pour le gérant, la marge brute par travailleur est beaucoup plus élevée. Il est en effet rémunéré sur une partie de la marge brute laitière totale (76 000 \$/an), l'autre partie étant redistribuée aux actionnaires sous forme de rémunération du capital de l'exploitation.

Ces performances montrent l'intérêt économique de ce type de fermes mécanisées dans lesquelles la productivité du travail est élevée. Cependant, la ferme IDP présente une productivité par hectare assez faible par rapport aux petites exploitations. Elle dégage une marge brute de seulement $6900 \$ /$ ha/an, ce qui s'explique par ses faibles performances fourragères (productivité de 20000 litres de lait / ha/an). Par ailleurs, comme pour les exploitations spécialisées de type 5 , ce modèle de grande ferme industrielle spécialisée apparaît plus vulnérable à des baisses du prix du lait.

Les performances économiques doivent aussi être évaluées d'un point de vue global, c'est-à-dire en prenant en compte les revenus issus des activités non-laitières. En effet, les exploitations diversifiées (types 1, 3 et 4) bénéficient, en plus du revenu laitier, de revenus complémentaires agricoles et non-agricoles. Ces revenus complémentaires représentent entre $35 \%$ et $45 \%$ du revenu d'exploitation et proviennent en moyenne de trois types d'activités différentes (cultures, élevage de monogastriques ou activités non-agricoles). Ainsi, les exploitations percevant les revenus les plus élevés ne sont pas forcément celles qui génèrent le revenu laitier le plus élevé. En l'occurrence, les exploitations de type 3 génèrent un revenu brut total du ménage de 16400 \$/ an, ce qui est très proche du revenu brut généré par les exploitations de type 2 et supérieur au revenu brut des exploitations de type 5 (tableau IV). Pourtant, la marge brute laitière de ces exploitations de type 3 est bien inférieure à celle des types 2 et 3 (tableau V). Ces différences illustrent l'importance de la pluriactivité dans la durabilité économique des exploitations.

En ce qui concerne la composante environnementale de la durabilité, les exploitations des types 2, 4 et 5 utilisent plus d'engrais (azote, phosphore, potassium) (figure 4 ; tableau V). Cependant, c'est surtout la ferme IDP qui utilise le plus d'intrants : elle consomme environ deux fois plus d'engrais et d'herbicides que les petites fermes. En outre, les exploitations peu ou moyennement intensives (types 1 et 3 ) sont autonomes en fourrages et présentent donc des performances environnementales intéressantes dans un contexte très contraint en ressources fourragères.

Du point de vue social, l'enquête souligne les différences en termes de charge de travail. Ces charges par travailleur sont plus élevées dans les exploitations ayant les plus grands cheptels (types 2 et 5). Cependant, le recours à des équipements (machine à traire et générateur) permet de réduire le temps de travail par bovin dans ces exploitations par rapport aux autres types où le travail est réalisé manuellement. Dans la ferme industrielle, le temps de travail sur l'atelier laitier des ouvriers est deux fois plus élevé que celui des exploitants familiaux, en raison du statut de salarié de ces employés qui travaillent huit heures par jour, sept jours sur sept, et 50 semaines par an. Dans les exploitations familiales, le temps de travail est comptabilisé uniquement pour l'atelier laitier. Or ces agriculteurs consacrent également une partie de leur temps aux autres activités d'élevage (porcs, volailles), agricoles (riz ou autres), voire non-agricoles.

\section{DISCUSSION}

\section{Facteurs locaux expliquant la diversité des exploitations laitières}

Cette étude a permis d'identifier cinq types d'exploitations laitières familiales. Ces types se différencient par leur capital physique, humain, social, naturel et financier, ainsi que par leurs performances économiques, environnementales et financières. Cette étude montre que le lait joue un rôle important dans les exploitations laitières à Ba Vi car il contribue à la valorisation financière du travail des familles. Le lait assure un revenu par hectare élevé et régulier à ces éleveurs, leur permettant de subvenir aux besoins de leur famille ainsi que d'investir dans leur exploitation (par exemple en achetant de nouveaux animaux). Par ailleurs, même si la production laitière est récente et si le modèle technique adopté par les éleveurs semble relativement homogène, les pratiques d'élevage ainsi que les performances techniques sont loin d'être identiques entre tous les éleveurs. Des suivis d'élevage pourraient ainsi être conduits pour rendre compte de la diversité, au sein de chaque groupe, des pratiques et des performances techniques, afin d'identifier des leviers d'amélioration utiles aux éleveurs (Hostiou et al., 2012).

Des facteurs historiques et sociaux expliquent en partie les différences identifiées en termes de dotation en capitaux entre les types. Pour la majorité de ces familles, le lait est une activité parmi d'autres. Seul le type 5 (exploitations spécialisées en lait et intensives, avec un troupeau laitier relativement grand) et la ferme industrielle sont spécialisés dans la production laitière. Les autres associent le lait à d'autres activités agricoles (porcs, volailles, cultures) et/ou non-agricoles (salariat, commerce). La pluriactivité est pratiquée dans les types ayant un cheptel de plus petite taille (types 1 et 4 ; encadré 1) car elle est source d'emploi et de revenus pour ces familles, et elle est favorisée par la proximité de la capitale Hanoï, dont les activités industrielles et touristique sont porteuses d'opportunités économiques (Duteurtre et al., 2015a).

Le lieu d'implantation des exploitations est également un facteur explicatif des différences observées. Les exploitations du type 5 sont localisées sur les terres du Centre de recherche en élevage de Ba Vi. Ces éleveurs sont souvent des ex-ouvriers de l'ancienne ferme d'Etat laitière, ayant reçu en fermage des terres et des animaux après la fermeture de cette ferme au début des années 1990. La grande taille des troupeaux observée aujourd'hui s'explique par cette histoire particulière.

L'ethnie est également un facteur expliquant les disparités entre les différents types d'exploitations. Ainsi, les fermes possédant les plus petits troupeaux appartiennent à des Muong qui pratiquent une 
agriculture plus traditionnelle, donc plus diversifiée et moins intensive, alors que les plus grandes exploitations sont gérées par des Kinh. Ces disparités ethniques dans le savoir-faire et la dotation en capital se retrouvent dans d'autres contextes au Vietnam (Van de Walle et Gunewardena, 2001).

Cette étude n'aborde pas spécifiquement les évolutions futures des exploitations laitières familiales mais elle soulève certaines perspectives. Si le modèle de ferme industrielle de grande taille se développe actuellement dans différentes régions du Vietnam (Nguyen Mai Huong et al., 2016), il ne constitue pas une perspective envisageable pour les fermes familiales. En effet, ce modèle de grande ferme industrielle nécessite des investissements importants en équipement et en cheptel, un accès à une emprise foncière significative, et des compétences en gestion. En l'occurrence, la ferme IDP, installée sur les terres de l'ancienne ferme d'Etat de Ba Vi, a bénéficié d'un partenariat étroit avec le centre de recherche. Des économies d'échelle sont aussi envisageables dans l'agriculture familiale, mais elles passent par des modèles d'organisation différents et par des solutions techniques spécifiques. Le développement de coopératives ou d'unions de producteurs pourrait par exemple permettre aux éleveurs familiaux d'accéder à des équipements collectifs afin d'améliorer la productivité du travail. L'amélioration des techniques fourragères, la pratique de l'ensilage, la mécanisation de la traite ou l'amélioration des revenus non-agricoles au sein de la famille pourraient aussi renforcer les capacités des petites fermes à se maintenir.

\section{Types d'exploitations laitières plus durables que d'autres?}

Cette étude permet de comparer certains aspects de la durabilité des cinq types d'exploitations laitières familiales et de la grande ferme industrielle identifiés à $\mathrm{Ba} \mathrm{Vi}$. Nos résultats permettent d'abord d'identifier les atouts et faiblesses de chacun des types. Ainsi, les marges brutes de l'atelier laitier des exploitations les plus intensives avec de grands troupeaux laitiers (types 2 et 5 ; encadré 1) sont meilleures que celles des autres types. Toutefois les exploitations pluriactives et de polyculture-élevage, qu'elles aient une grande surface agricole (types 1 et 3) ou non (type 4), sont capables de compenser une éventuelle baisse du prix du lait grâce à la présence de multiples activités (Udo et al., 2011). Elles sont donc plus flexibles.

De plus, ces exploitations pluriactives ont des performances environnementales plus intéressantes. D'une part, certaines utilisent moins d'engrais et d'herbicides que les exploitations les plus intensives (type 2 et ferme IDP) et seraient donc potentiellement moins polluantes. Les résultats ont souligné les impacts potentiels négatifs de l'élevage industriel sur la qualité des sols et de l'eau, liés à la gestion intensive des fourrages sur cette grande ferme laitière. Il conviendrait évidemment de confirmer cela par des analyses environnementales plus poussées. D'autre part, certaines sont autonomes en fourrages. Enfin, les interactions positives entre cultures et élevage limitent la pollution locale car les effluents d'élevage sont valorisés sur les parcelles cultivées (Herrero et al., 2010). Du point de vue social, l'efficacité du travail (nombre d'heures par bovin par an) est meilleure dans les types 2 et 5 grâce aux équipements (machine à traire, générateur électrique), mais leurs charges de travail sont plus élevées.

Cette étude ne permet pas de conclure qu'un type d'exploitation est plus durable que d'autres. Aucun type d'exploitation n'est meilleur que les autres pour l'ensemble des dimensions de la durabilité. Nos résultats montrent plutôt qu'il existe plusieurs compromis possibles entre revenus financiers, flexibilité de l'exploitation, impact environnemental et impact social, et qu'il est difficile d'arbitrer entre ces compromis.

Par ailleurs, les indicateurs utilisés dans cette étude pour évaluer la durabilité des exploitations laitières sont exprimés essentiellement à l'échelle de la ferme. Or, la durabilité des exploitations laitières est aussi fortement impactée par les liens à la filière, et aux autres acteurs et activités du territoire (Napoléone et al., 2015). Ainsi, cette étude pourrait être complétée par des travaux visant à évaluer des indicateurs de durabilité prenant en compte les interactions entre l'exploitation, la filière et le territoire (Vilain et al., 2008 ; Lebacq et al., 2013).

\section{Quels modèles laitiers et quelles capacités à accroître les volumes produits?}

La stratégie nationale de développement de l'élevage au Vietnam vise à développer l'élevage laitier sur la base du modèle des grandes exploitations laitières intensives. Les types 2 et 5 , et surtout la ferme IDP, constituent les modèles d'exploitations laitières dont le développement est encouragé. Cependant, notre étude montre que les fermes avec de petits cheptels (types 1, 3 et 4) ont également un rôle à jouer dans le développement de la production laitière nationale : elles sont moins vulnérables aux fluctuations du prix du lait du fait d'un revenu basé sur une diversité d'activités agricoles et/ou non-agricoles, et leurs performances environnementales et sociales sont plus intéressantes. Ainsi, les performances économiques des exploitations agricoles ne devraient pas être le seul indicateur à prendre en compte dans les politiques nationales, dès lors que l'on chercherait à promouvoir un développement durable de l'élevage au Vietnam. Par exemple, les exploitations du type 5 localisées sur les terres du centre de recherche ne possèdent pas de certificat de droit d'usage de leurs terres, ce qui ne leur permet pas d'emprunter de l'argent auprès de banques, comme le requiert la législation vietnamienne.

Ces résultats nous amènent à proposer quelques pistes pour accompagner le développement de l'activité laitière au Vietnam. Des mesures pourraient être mises en place pour maintenir un tissu d'exploitations laitières diverses quant à leur taille, l'utilisation de la main d'œuvre et les combinaisons d'activités agricoles et non-agricoles. En effet, les voies d'accroissement des volumes de lait produits sont à adapter à chaque type d'exploitation. Les fermes de polyculture-élevage et pluriactives (types 1 et 4 ) pourraient accroître la quantité de lait produite par l'augmentation du cheptel et l'investissement dans des équipements (machine à traire), et donc par l'accès à des prêts à faible taux d'intérêt. L'augmentation de la production est plus difficile pour le type 3 à cause du foncier limité et de faibles capacités financières. Les exploitations intensives avec de grands troupeaux laitiers (types 2 et 5) peuvent difficilement accroître les volumes produits par l'augmentation du cheptel, mais elles le pourraient par l'amélioration génétique des vaches et l'augmentation du rendement en fourrages. La charge de travail est une contrainte dans les exploitations avec de petits cheptels pour accroître les volumes de lait en raison du faible niveau de mécanisation de la traite ou encore de l'affouragement, ces travaux se faisant essentiellement manuellement (Hostiou et al., 2012). Pour les exploitations avec de petits cheptels et/ou avec de faibles capacités financières, il faudrait pouvoir développer une mécanisation en commun, comme cela est également envisagé dans d'autres pays du Sud (Chand et al., 2015). L'organisation en coopératives ou en groupes d'intérêts plus informels pourrait se révéler pertinente, mais du fait de l'histoire du collectivisme dans ce pays des modalités politiques et sociales sont à prendre en compte.

\section{CONCLUSION}

Cette étude caractérise la diversité et la durabilité des exploitations laitières au Vietnam, dans un contexte où les politiques publiques privilégient l'accroissement de la taille des cheptels, la spécialisation et l'intensification des exploitations, afin d'augmenter rapidement les volumes produits. Si des exploitations laitières spécialisées de grande taille se développent, la production repose également sur des exploitations mixtes d'agriculture-élevage et sur des ménages ruraux 
pluriactifs. Les exploitations de grande taille et les fermes les plus intensives présentent des performances économiques (marge brute totale de l'atelier laitier) plus élevées que celles des exploitations de plus petite taille et diversifiées. Cependant, les exploitations avec de petits cheptels ont également des atouts sur le plan économique (vulnérabilité moindre à des fluctuations du prix du lait par exemple), environnemental (complémentarités agriculture-élevage, autonomie fourragère, utilisation moindre d'engrais) et social (emploi rural) et présentent donc un intérêt pour le développement durable de la production laitière nationale. Le développement de la production laitière à $\mathrm{Ba} \mathrm{Vi}$, et d'une manière générale au Vietnam, pourrait reposer sur le maintien de cette diversité d'exploitations avec la mise en place de mesures qui traiteraient différemment, pour chaque type d'exploitation, des problèmes d'accès à la main d'œuvre, à la mécanisation, au crédit ou encore au foncier. Ces résultats pourraient être discutés avec des décideurs politiques locaux et nationaux afin d'éclairer les trajectoires de développement de l'élevage laitier dans les régions laitières du Vietnam.

\section{REFERENCES}

Agroinfo, 2014. Rapport de la filière laitière, 51 p.

Beghin J.C., 2006. Evolving dairy markets in Asia: Recent findings and implications. Food Policy, 31: 195-200, doi: 10.1016/j. foodpol.2006.03.001

Bosc P.M., Sourisseau J.M., Bonnal P., Gasselin P., Valette E., Belieres J.F., Friedmann H., 2014. Diversité des agricultures familiales, exister, se transformer, devenir. Quae, Versailles, France, 308 p.

Brossier J., Marshall E., Chia E., Petit M., 2003. Gestion de l'exploitation agricole familiale. Eléments théoriques et méthodologiques. Educagri, Dijon, France, 220 p.

Carney D., Drinkwater M., Rusinow T., Neefjes K., Wanmali S., Singh N., 1999. Livelihoods approaches compared. A brief comparison of the livelihoods approaches of the UK Department for International Development (DFID). CARE, Oxfam, UN Development Programme, London, UK

Chambers R., Conway G., 1992. Sustainable rural livelihoods: practical concepts for the 21 st Century. Institute of Development Studies, Brighton, UK (Discussion Paper 296)

Chand P., Sirohi S., Sirohi S.K., 2015. Development and application of an integrated sustainability index for small-holder dairy farms in Rajasthan, IndiaPrem. Ecol indic, 56: 23-30, doi: 10.1016/j. ecolind.2015.03.020

Dray S., Dufour A.B., Chessel D., 2007. The ade4 package-II: Two-table and K-table methods. $R$ News, 7: 47-52

Duteurtre G., Cesaro J.D., Nguyen Mai Huong, Pham Duy Khanh, Nguyen Ngoc Luan, 2015b. The TH dairy company (Vietnam): Is such a large-scale investment sustainable? Report of a visit conducted in Nghĩa Dàn District on 2 July 2015. Cirad, Rudec, Hanoi, Vietnam, 17 p. (Revalter Working Paper) www.futurelivestock.net/index. php? language $=1 \&$ module $=$ document $\&$ cateld $=1290$

Duteurtre G., Faye B., 2009. L'élevage, richesse des pauvres. Stratégies d'éleveurs et organisations sociales face aux risques dans les pays du Sud. Quae, Versailles, France, 288 p.

Duteurtre G., Pham Duy Khanh, Cesaro J.C., 2015a. Bassin laitier de $\mathrm{Ba} \mathrm{Vi}$ : un territoire d'élevage façonné par les politiques publiques, entre modèle industriel et soutien à la paysannerie. In : Voies lactées. Dynamique des bassins laitiers entre globalisation et territorialisation (éds Napoléone M., Corniaux C., Leclerc B.). Cardère, Paris, France, 67-88

Escofier B., Pages J., 1994. Multiple factor analysis (AFMULT package). Comput. Stat. Data Anal., 18: 121-140

GSO, 2014. Results of the 2011 Rural, agricultural and fishery census, General Statistical Office, Hanoi, www.gso.gov.vn/default_ en. aspx?tabid=477\&ItemID=13399 (accessed 3 March 2016)

Gura S., 2008. Industrial livestock production and its impact on smallholders in developing countries. Consultancy report to the League for Pastoral Peoples and Endogenous Livestock Development, www.pastoralpeoples.org (accessed 23 Feb. 2016)

Hemme T., Otte J., 2010. Status and prospects for smallholder milk production. A global perspective. FAO, Rome, Italy, $186 \mathrm{p}$.

Herrero M., Thornton P.K., Notenbaert A.M., Wood S., Msangi S., Freeman H.A., Bossio D., et al., 2010. Smart investments in sustainable food production: revisiting mixed crop-livestock systems. Science, 327: 822-825, doi: 10.1126/science. 1183725
Hostiou N., Pham Duy Khanh, Duteurtre G., Vu Trong Binh, Dedieu B., 2012. Relationships between work organisation and size of dairy farms: a case study based in Vietnam. Trop. Anim. Health Prod., 44: 1709-1716, doi: 10.1007/s11250-012-0128-y

Lairez J., Feschet P., Aubin J., Bockstaller C., Bouvarel I., 2015. Agriculture et développement durable. Guide pour l'évaluation multicritère. Quae, Versailles, France, 232 p.

Lebacq T., Baret P.V., Stilmant D., 2013. Sustainability indicators for livestock farming. A review. Agro Sustain. Dev., 33: 311-327, doi: 10.1007/s13593-012-0121-x

López-Ridaura S., Masera O., Astier M., 2002. Evaluating the sustainability of complex socio-environmental systems. The MESMIS framework. Ecol. Indic., 2: 135-148, doi: 10.1016/S1470160X(02)00043-2

Napoléone M., Corniaux C., Leclerc B., éds, 2015. Voies lactées. Dynamiques des bassins laitiers entre globalisation et territorialisation. Cardère, Avignon, France, 313 p.

Ness B., Urbel-Piirsalu E., Anderberg S., Olsson L., 2007. Categorising tools for sustainability assessment. Ecol. Econ., 60: 498-508, doi: 10.1016/j.ecolecon.2006.07.023

Nguyen Mai Huong, Duteurtre G., Pham Duy Khanh, Hoang V.Q., Cesaro J.C., 2016. Hướng đi nào b ên vững cho phát triển chăn nuôi bò sữa ở Việt Nam: sản xuất nông hộ hay đầ tư qui mô lớn? Tạp chí Khoa học và Công nghệ chăn nuôi, Viện chăn nuôi Quốc gia, Số, 61: 12-21. (Quels sont les modèles durables pour l'élevage laitier du Vietnam : I'exploitation laitière familiale ou le méga ferme ? Revues des scientifiques et de la technologie de l'élevage - Institut national d'élevage du Vietnam)

OECD, 2015. Agricultural Policies in Viet Nam 2015. Organisation for Economic Cooperation and Development, Paris, France

Salas-Reyes I.B., Arriaga-Jordan C.M., Rebollar-Rebollar S., GarciaMartinez A., Albarran-Portillo B., 2015. Assessment of the sustainability of dual-purpose farms by the IDEA method in the subtropical area of central Mexico. Trop. Anim. Health Prod., 47: 1187-1194, doi: 10.1007/s11250-015-0846-z

Steinfeld H., Mooney H.A., Schneider F., Neville L.E., Eds, 2010. Livestock in a changing landscape, Vol. 1, Drivers, consequences and responses. Island Press, Washington DC, USA, $416 \mathrm{p}$.

Suzuki K., Kanameda M., Ogawa T., Nguyen T.T.D., Dang T.T.S., Luu Q.H., Pfeiffer D.U., 2006. Productivity and socio-economic profile of dairy cattle farmers amongst rural smallholder communities in northern Vietnam. Livest. Sci., 101: 242-250, doi: dx.doi. org/10.1016/j.livprodsci.2005.11.015

Udo H.M.J., Aklilu H.A., Phong L.T., Bosma R.H., Budisatria I.G.S., Patil B.R., Samdup T., Bebe B.O., 2011. Impact of intensification of different types of livestock production in smallholder croplivestock systems. Livest. Sci., 139: 22-29, doi: dx.doi.org/10.1016/j. livsci.2011.03.020

Van de Walle D., Gunewardena D., 2001. Sources of ethnic inequality in Viet Nam. J. Dev. Econ., 65: 177-207

Vilain L., Girardin P., Mouchet C., Viaux P., Zahm F., 2008. La méthode IDEA, indicateurs de durabilité des exploitations agricoles : guide d'utilisation, vers. 3. Educagri, Dijon, France, www.idea.chlorofil.fr/ (consulté 30 mars 2014) 


\section{Summary}

Pham Duy Khanh, Duteurtre G., Cournut S., Messad S., Dedieu B., Hostiou N. Characterizing dairy farm diversity and sustainability in Vietnam: Case study in suburban Hanoi

In Vietnam, public policies tend to support the development of large (> 30 cows) or industrial (> 500 cows) dairy farms. However, despite the recent expansion of these large farms, very small family farms still play a major role in national milk production. To contribute to the discussion on the models of farms worth promoting, we aimed to characterize the diversity of dairy farms in the district of Ba Vi in suburban Hanoi, and to assess their sustainability. In total 160 dairy farms were selected to represent the diversity of farms in the area. Directional interviews were conducted with farmers on the various types of capital available on the farm, on livestock-husbandry and milk-marketing practices, and on farm economic, environmental and social performances. A multiple factor analysis, followed by hierarchical clustering enabled us to establish a farm typology. An industrial farm and five types of family dairy farms were identified in the district, mainly differentiated by the number and type of agricultural and non-agricultural activities, the dairy-herd size, and milk production intensification. The performance indicators of the specialized farms revealed higher economic profitability than in more diversified farms. However, mixed farms also had economic (adaptive flexibility), environmental (fodder autonomy) and social (employment, workload) assets that were related to the diversity of their agricultural and non-agricultural activities. Although policies tend to support the development of specialized farms and large farms, support programs must also include small farms with agricultural and non-agricultural activities, as they also play an essential role in the sustainable development of national dairy production.

Keywords: dairy farm, diversification, intensification, typology, Viet Nam

\section{Resumen}

Pham Duy Khanh, Duteurtre G., Cournut S., Messad S., Dedieu B., Hostiou N. Caracterización de la diversidad y sostenibilidad de fincas lecheras en Vietnam: Estudio de un caso en los suburbios de Hanoi

En Vietnam, las políticas públicas tienden a respaldar el desarrollo de fincas lecheras grandes (> 30 vacas) o industriales (> 500 vacas). Sin embargo, a pesar de la reciente expansión de estas fincas grandes, fincas familiares muy pequeñas todavía ocupan un papel mayor en la producción doméstica de leche. Con el fin de aportar a la discusión sobre los modelos de fincas que vale la pena promover, pretendemos caracterizar la diversidad de las fincas lecheras en el distrito de Ba Vi en los suburbios de Hanoi y asesorar su sostenibilidad. Se seleccionaron 160 fincas lecheras para representar la diversidad de fincas en el área. Se condujeron entrevistas direccionales con los finqueros sobre los diversos tipos de capital accesible en la finca, sobre las prácticas de cría-ganadería y mercadeo de la leche y sobre los rendimientos económico, ambiental y social de la finca. Un análisis multifactorial, seguido de un clustering jerárquico nos permitió establecer una tipología de las fincas. Se identificaron un tipo de finca industrial y cinco tipos de fincas lecheras familiares en el distrito, principalmente diferenciadas por el número y tipo de actividades agrícolas y no agrícolas, el tamaño del hato lechero y la intensificación en la producción de leche. Los indicadores de rendimiento de las fincas especializadas revelaron una rentabilidad más alta que en las fincas más diversificadas. Sin embargo, las fincas mixtas también presentaron valores económicos (flexibilidad adaptable), ambientales (autonomía de forraje) y sociales (empleo, carga de trabajo), que estaban relacionados con la diversidad de sus actividades agrícolas y no agrícolas. A pesar de que las políticas tienden a apoyar el desarrollo de fincas especializadas y fincas grandes, los programas de apoyo deben también incluir fincas pequeñas, con actividades agrícolas y no agrícolas, ya que ellas juegan un rol esencial en el desarrollo sostenible de la producción lechera nacional.

Palabras clave: granja lechera, diversificación, intensificación, tipología, Vietnam 
